\title{
Origin of homochirality in biological systems
}

\author{
S. Toxvaerd \\ Department of Chemistry, H. C. Ørsted Institute, DK-2100 Copenhagen Ø, Denmark \\ e-mail:tox@st.ki.ku.dk
}

\begin{abstract}
Models for segregation of racemic mixtures of chiral amphiphiles and lipophiles in aqueous solutions show that the amphiphiles with active isomerization kinetics can perform a spontaneous symmetry break during the segregation and self-assemble to homochiral matter. Based on physicochemical facts, it is argued that D-glyceraldehyde, which was synthesized from the volatiles at the hydrothermal reactors at the origin of life, could be the origin of homochirality in biological systems. Received 14 March 2005, accepted 12 April 2005
\end{abstract}

Key words: D-glyceraldehyde, homochirality, origin of life.

\section{Introduction}

Biological systems can be characterized as soft condensed and stereospecific matter. Even the simplest bacteria have membranes with homochiral molecules and a metabolism and cytokinesis where the stereospecific interactions are the common feature. The stereospecific biochemical reactions take place in an aqueous environment. The L-amino acids and D-sugar molecules constitute the basic building blocks in all living systems and it is not obvious how this homochirality has originated, nor how it has been maintained, since aqueous solutions of L-amino acids or D-sugars will racemize due to active isomerization kinetics and lose their homochiral order within a relatively short time: for a diluted aqueous solution of a simple triose this is within a day at $70-100{ }^{\circ} \mathrm{C}$ (Fedoroňko \& Königstein 1969) in alkaline conditions. For L-amino acids, it takes much longer (Bada 1972), but is still a very short time compared with the time it must have taken to evolute a coli-bacteria with very complex stereospecific biochemical machinery. It is therefore natural to ask whether homochirality is a precondition for biological evolution, or whether the self-assembling of the biochemical molecules at the origin of life, if started from racemic mixtures, generated the homochirality during the evolution.

Inspired by Pasteur's (1850) famous experiment many assume that the bio-homochirality was obtained at solid surfaces or by crystallization (Avalos et al. 2004), but the route to a micelle assembly or an assembly of primitive vesicles with biochemical components seems to go via (diluted) aqueous solutions which, as mentioned above, destroy the homochiral order. It is therefore challenged to look for a possible biomolecular chiral candidate and a mechanism for obtaining homochirality of ensembles of this molecule directly in the fluid state. Clearly one needs a molecule which has a strong energetic preference for interactions with a molecule with the same configuration, rather than with a molecule with the mirror configuration. This is due to the fact that, in order to generate homochiral clusters, it has to overcome the entropy of mixing in the aqueous solution and/or the entropy of mixing between the two enantiomer configurations in a droplet.

The difference in stereospecific interactions is called chiral discrimination and it can both be in favour of homochiral arrangements (D-D and $\mathrm{L}-\mathrm{L}$ ) for some molecules as well as the racemic arrangement (D-L) for other molecules. However, even when the chiral discrimination favours a homochiral arrangement, as was the case in Pasteur's experiment, the clustering only ensures local homochirality. In the case of homochirality by crystallization, one gets both kinds of crystal unless one has a mechanism which breaks the symmetry (Kondepudi et al. 1990). It is here that the isomerization kinetics play a crucial role. It is not active in crystals, but is active in the fluid state and at high temperature, especially in the keto-enol isomerization kinetics for sugar. It is these kinetics that ensure racemic solutions at low chiral concentrations, which might also ensure homochirality at high concentrations. At low concentrations of chiral molecules there is no clustering and the kinetics are observed to drive the solution to a racemic mixture even if one starts with, e.g., pure L-amino acids. For this reason one has to look for physico-chemical conditions of mixtures with a high concentration of chiral molecules with a strong chiral discrimination in favour of homochirality.

\section{Domain catalysed isomerization kinetics}

A model system was set up in order to investigate the role of isomerization kinetics in condensed fluid systems with a high concentration of chiral molecules (Toxvaerd 2000, 2004a). The model system simulated (molecular dynamics), in a 
qualitative way, fluid mixtures of molecules with discrimination in favour of homochiral clustering. In a simple qualitative model of Lennard-Jones (LJ) particles, which does not explicitly account for interactions such as hydrogen bonds, the chiral discrimination can be obtained by varying the attraction between pairs of $\mathrm{D}, \mathrm{D}(=\mathrm{L}, \mathrm{L})$ and $\mathrm{D}, \mathrm{L}$ (Toxvaerd 2000). The strength of this chiral discrimination can be estimated from the difference in the thermodynamic energy, $U(T, V)$, between a homochiral fluid and a racemic mixture, and it was $\approx 1 \mathrm{~kJ} \mathrm{~mol}^{-1}$.

The isomerization kinetics are active in the fluid state and in an aqueous solution especially at high temperatures. The kinetics for the conversion

$\mathrm{D}+\mathrm{D} \underset{E_{\mathrm{DL}}}{\stackrel{E_{\mathrm{DD}}}{\rightleftharpoons}} \mathrm{D}+\mathrm{L} \underset{E_{\mathrm{LL}}}{\stackrel{E_{\mathrm{DL}}}{\rightleftharpoons}} \mathrm{L}+\mathrm{L}$

were implemented, e.g., as collision activated kinetics with activation energies $E_{\mathrm{DD}}, E_{\mathrm{LL}}$ and $E_{\mathrm{DL}}$. The activation energy, $E_{\mathrm{DL}}$, for DL-collisions, which may convert a D-molecule into a L-molecule or vice versa, must be less than the corresponding activation energy, $E_{\mathrm{DD}}=E_{\mathrm{LL}}$ allowing a conversion of one of the molecules in the collisions. This inequality

$E_{\mathrm{DL}}<E_{\mathrm{DD}}=E_{\mathrm{LL}}$

accounts for the chiral discrimination with a lower potential energy of pairs and domains of enantiomers. The actual values of the activation energies used in the simulations (with $\varepsilon / k=500 \mathrm{~K}$ ) were $E_{\mathrm{DD}}=5 \varepsilon \approx 20 \mathrm{~kJ} \mathrm{~mol}^{-1}$ and $E_{\mathrm{DL}}=3 \varepsilon$ $\approx 12 \mathrm{~kJ} \mathrm{~mol}^{-1}$. The activation energy for the corresponding isomerization kinetics for a simple triose, glyceraldehyde, in the diluted regime where $E_{\mathrm{DD}}=E_{\mathrm{LL}}=E_{\mathrm{DL}}$ is $18.7 \mathrm{~kJ} \mathrm{~mol}^{-1}$ (Fedoroňko \& Königstein 1969).

The system was bistable at high concentrations due to the isomerization kinetics and the kinetics resulted in a dominance of one of the species at late times.

The time evolution of systems of many thousands of Dand L-particles, started as racemic fluid and/or concentrated solute mixtures, was obtained by molecular dynamics. For computational details see Toxvaerd (2000, 2004a). Figure 1 shows examples of symmetry breaks in racemic mixtures (of 40000 particles), given by the excess fraction between $\mathrm{D}$ - and L-particles. Ensembles of $\approx 10$ simulations for each set of activation energies and sufficient strengths of the chiral discrimination all gave a symmetry break, and it was not possible to predict when the symmetry breaks appeared or which of the two types would dominate at late times. However, it was usually the largest of the established homochiral subdomains in the fluid or the largest homochiral droplet in the solution that finally dominated. The systems ended in the equilibrium states with (for, e.g., a D-particle dominance)

$\frac{[\mathrm{D}]}{[\mathrm{L}]}=\exp \left(-\frac{\left(E_{\mathrm{DL}}-E_{\mathrm{DD}}\right)}{k T}\right)$

in accordance with kinetics theory. (The pre-exponential collision parameter, $A$, in the Arrhenius expression is the same for the two kinds of conversions in the simple model.)

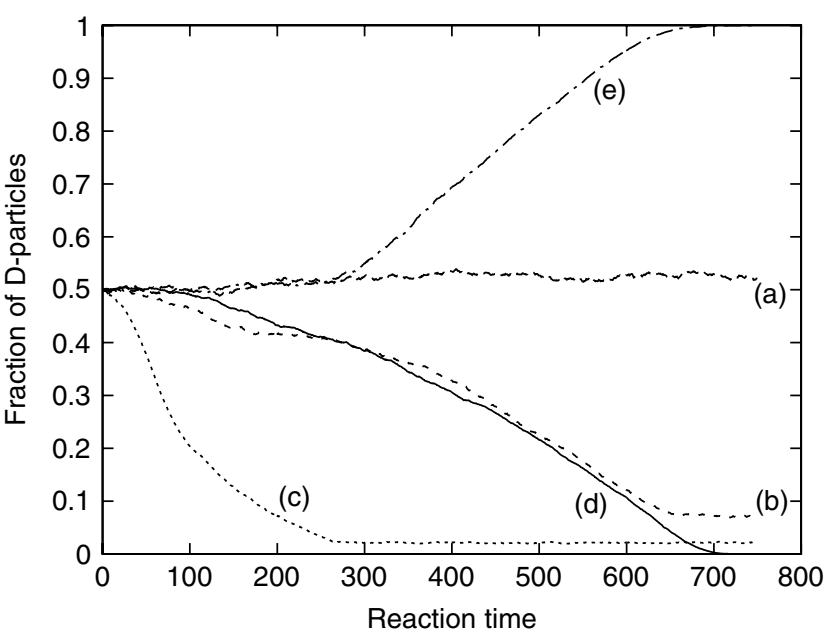

Fig. 1. Fraction of D-particles as a function of reaction time in reduced units (one reduced time unit $\approx 2 \mathrm{ps}$ ) and for different strengths of chiral discrimination and activation energies (Toxvaerd 2000). The system remains racemic when there is only a small difference in activation energies, $E_{\mathrm{DD}}=E_{\mathrm{LL}} \leqslant E_{\mathrm{DL}}+k T$ (a); but the kinetics result in symmetry breaks (b)-(d) for larger differences and it gives a dominance of one of the enantiomers at late times.

Biochemical molecules can be divided into hydrophilic, amphiphilic and lipophilic molecules. It is natural to believe that all three types have been synthesized in huge amounts at the origin of life. Amphiphiles can separate together with hydrophobic compounds in water in various ways including emulsion-like separation and micelle formation. A simple qualitative model for phase segregation in a ternary mixture was set up (Toxvaerd 2004b) in order to investigate emulsionlike segregation in aqueous solutions. The segregation was obtained by cooling down the mixture, whereby the hydrophobic particles segregated in droplets. The amphiphilic particles segregate together with the hydrophobic particles into droplets and with some of the amphiphilic particles located in the droplet interphase in a surfactant-like manner. The amphiphilic particles segregated with the hydrophobic particles into droplets, but with the amphiphilic particles also spread in the droplet interface in a surfactant-like manner. Figure 2 shows the distribution of the amphiphilic particles in a droplet of amphiphilic and hydrophobic particles (hydrophobic and water particles are not shown) after an emulsionlike segregation.

The possibility of an emulsion-like separation with a sub-domain containing only the amphiphiles is important for the stereospecific ordering described in (Toxvaerd 2000, 2004a). If a multicomponent mixture of organic components including the amphiphiles is cooled down and performs this kind of phase segregation, the amphiphiles can undergo an ordering into one of its enantiomer configurations by the domain-catalysed isomerization kinetics of the molecules in the enriched sub-domain. At the same time, the amphiphiles are present in the interface between the hydrophobic components and water, where they can act as the 


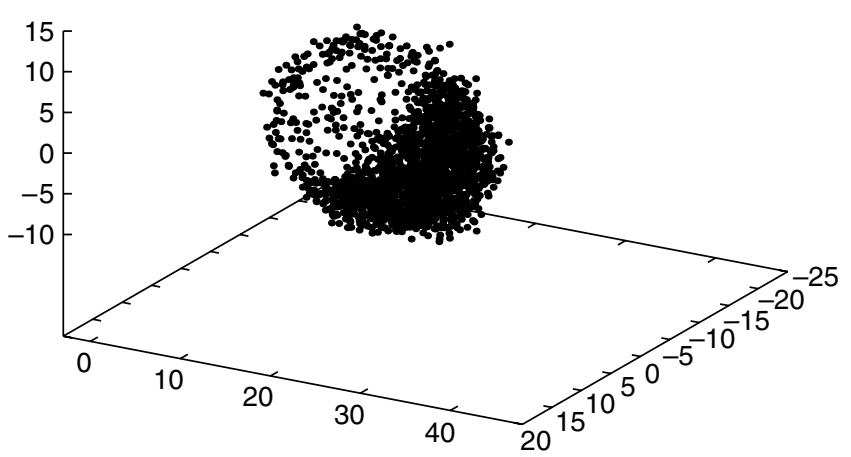

Fig. 2. A droplet of amphiphilic and hydrophobic particles segregated from an aqueous solution by cooling down the system. Only the amphiphiles are shown.

template and source for the synthesis of stereospecific biomolecules.

\section{Glyceraldehyde}

The model for domain-autocatalytic enhancement of homochirality in condensed fluid systems can, in principle, be applied to many simple chiral molecules in biological evolution. In locating a molecular candidate for homochirality obtained by these kinetics and the conditions for the symmetry break, one has the following criteria to look for.

(1) The molecule should first of all exhibit a strong chiral discrimination in favour of homochirality.

(2) It should have active isomerization kinetics at relevant temperatures and pressure.

(3) It should not be very soluble in water because it almost unavoidably would give a diluted aqueous solution, where one knows that it will be maintained in a racemic proportion.

(4) It should have been present in huge amounts at the origin of life.

(5) If the origin of life started with obtaining homochiral domains of stereo-specific molecules, one would expect these molecules to be central for the succeeding synthesis and self-assembly of stereospecific bio-molecules. For this reason one would expect the molecule(s) to show up in bio-systems today as 'key' molecule(s).

(6) Finally one needs a constrained environment which is able to maintain the biochemical components in the emulsion at sufficient high concentrations.

The second criterion points towards a sugar molecule, rather than an amino acid, because the keto-enol kinetics is much faster than the substitution isomerization kinetics for amino acids. However, simple sugar molecules are generally very solvable in water due to the one-to-one ratio between the number of hydrophilic functional groups and number of carbon atoms. There is, however, a spectacular exception: the simple triose glyceraldehyde (GLA; see Fig. 3), $\mathrm{CH}_{2} \mathrm{OH}-$ $\mathrm{CHOH}-\mathrm{CHO}$, is not very solvable in water $(3 \mathrm{~g}$ in $100 \mathrm{ml}$ at $20{ }^{\circ} \mathrm{C}$ ), nor in a hydrophobic solvent (Toxvaerd 2004b). The

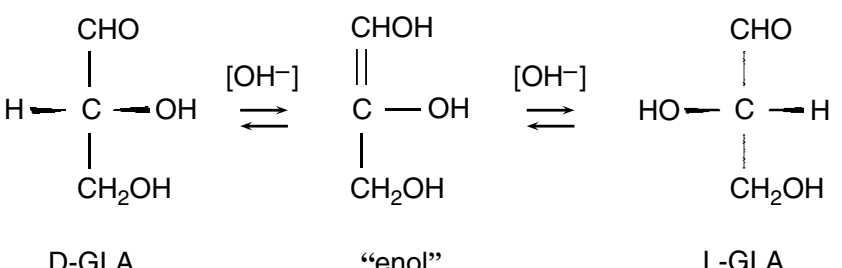

Fig. 3. Glyceraldehyde and its isomerization kenetics.

isomerization kinetics are fast and catalysed by $\left[\mathrm{OH}^{-}\right]$ (Fedoroňko \& Königstein 1969).

In addition, it has the strongest chiral discrimination the present author is aware of. The strength of chiral discrimination shows up in the difference in melting-point temperatures between the homochiral crystal and the racemic crystal. A strong chiral discrimination in favour of homochiral crystals results in a higher melting-point temperature of the homochiral crystal than the racemic. The homochiral GLA crystal melts at $194-198^{\circ} \mathrm{C}$ (Morgenlie 1973), whereas the racemic crystal melts at a significantly lower temperature of $130-132{ }^{\circ} \mathrm{C}$ (Gresham \& Grigsby 1949; Vik 1974), which gives a temperature difference of $\approx 65^{\circ} \mathrm{C}$ in a strong favour of the enantiomer molecular arrangement. (The chiral discrimination in the crystalline phase generally favours the racemic crystal (Leclercq et al. 1976) and none of the systems investigated there a temperature difference in favour of homochirality of more than $\approx 25^{\circ} \mathrm{C}$.)

In conclusion, GLA fulfils the three first criteria, but it also fulfils the next two. GLA was synthesized in the formose reaction, which is believed to be the source of sugar and related materials at the origin of life. The formose reaction is the spontaneous condensation of formaldehyde into sugar, which was discovered by Butlerow (1861), and the aldol-like mechanism is described by Breslow (1959). The two first steps are shown in Fig. 4, which gives a racemic mixture of D-GLA and L-GLA. Formaldehyde, $\mathrm{CH}_{2} \mathrm{O}$, is the simplest carbohydrate and it can be synthesized from carbon dioxide. It is produced in a reducing atmosphere containing $\mathrm{CO}_{2}$ and methane (Schlesinger \& Miller 1983), but what is more interesting it is also synthesized from volcano volatiles by reduction with either methane, hydrogen or sulfide minerals (Vladimirov et al. 2004). The condensation into sugar is not only catalysed by amino acids (Weber 2001), but also by naturally occurring aluminosilicates at the so-called black smokers or hydrothermal springs (Gabel \& Ponnamperuma 1967). So the formose reaction is well known and is believed to be the source of sugars and related bio-organic molecules at the origin of life (Gabel \& Ponnamperuma 1967; Washington 2000).

GLA also fulfils criterion 5. D-GLA-3-phosphate is a central molecule in glycolysis, and glycolysis only requires (phosphorylated) sugar for the spontaneous reaction, all of which can be obtained from the formose reaction, deep down in the oceans. Glycerol, the reduced form of GLA, is the 'anchor' molecule in membrane molecules so the homochirality of the anchor molecules in the membranes and the 
<smiles>CCOCCC(O)C=O</smiles><smiles>CCO[CH2+]C(C=O)C=CC(O)(C=O)CO</smiles>

Fig. 4. The two first steps in the formose reaction, which gives a racemic mixture of GLA.

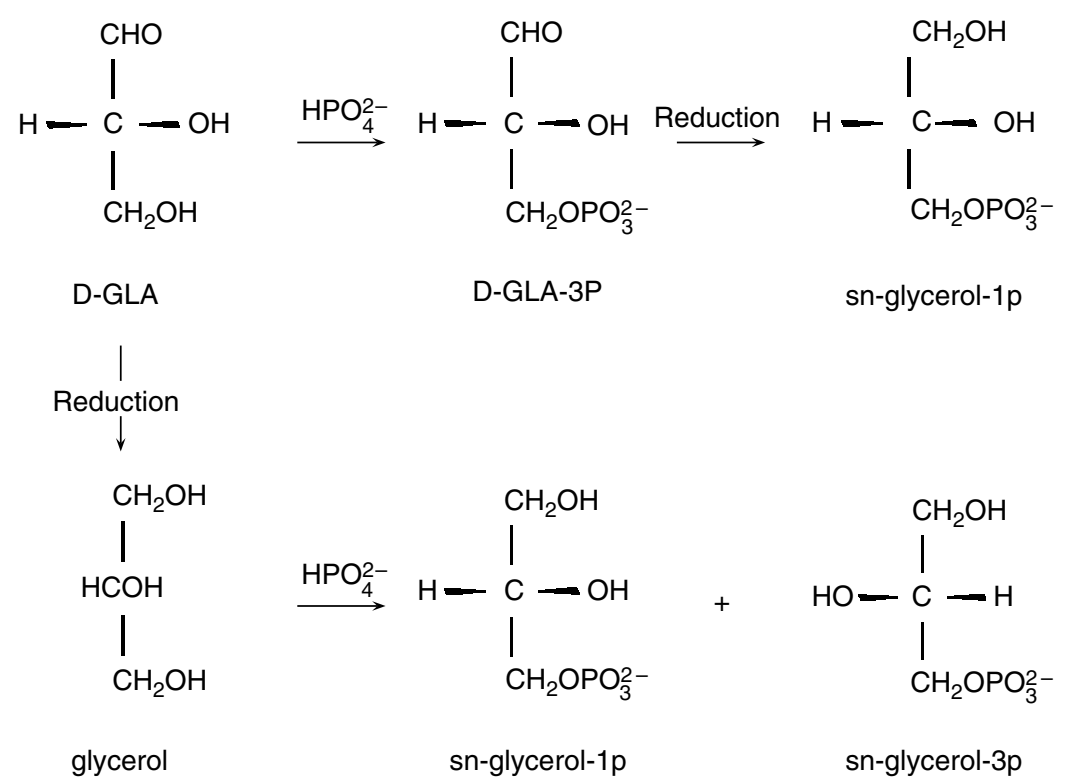

Fig. 5. sn-glycerol-1-phosphate and sn-glycerol-3-phosphate synthesized from D-GLA (note that due to IUPAC nomenclature rules the reduced product of D-GLA-3P is sn-glycerol-1-phosphate).

central position of D-GLA-3-phosphate in the basal metabolism could be the track laid by the biological evolution of the origin of homochirality in life.

\section{Discussion}

Around 4 billion years ago, the ocean was believed to be alkaline and reducing. This is known as the Hadean ocean, and one of the hypotheses about the origin of life is that it occurred in this ocean in 'black smokers' of hydrothermal reactors in compartments (Martin \& Russell 2003). There are many experimental observations which support this hypothesis (Martin \& Russell 2003; Russell \& Martin 2004). The syntheses of many of the central biochemical molecules from the volatiles at the black smokers are, in general, exergonic (Shock 1992; Amend \& Shock 2001; Weber 2002) under alkaline conditions. The volatiles of methane, hydrogen and carbon dioxide must have created a pool of simple biochemical molecules with Gibbs free energies favourable for a spontaneous synthesis and self-assembly of bio-systems (Davis 1998; Amend \& Shock 2001). The pool of biomolecules most likely also contained the ingredients for spontaneous creation of esters and ethers in the membranes (Weber 1991) and glyceraldehyde and glycolaldehyde are believed to have played a central role in the synthesis of biomolecules (Weber 1987, 1992, 1997). The environment at the hydrothermal reactors fulfils the last condition set up in the previous section.

A common feature for all living systems, in addition to glycolysis, is the homochiral molecules in the membranes, but with a notable difference. Archaean membrane molecules consist of (ether) derivatives of sn-glycerol-1-phosphate, whereas the molecules in the membranes of Bacteria and Eucarya consist of (ester) derivatives of sn-glycerol-3-phosphate. The membrane molecules are now synthesized by enzymes which, however, have no common sequences, and 
an analysis of these enzymes in different Archaea and Bacteria indicates that there is no common enzyme ancestor (Koga et al. 1998). In a living organism the membrane building blocks are synthesized from, e.g., glycerol with help of these stereospecific enzymes (Peretó et al. 2004), but at the origin of life we must search for a reaction pathway of spontaneous self-assembly from the ingredients in the compartments at the black smokers. There are two facts, which together are notable: all of the elementary membrane molecules in a cell are homochiral and all of these molecules are derivatives with a central chiral chain of three carbon atoms. It is notable because one can obtain simple vesicles with membranes from amphiphiles with only one or two central carbon atoms and with only one hydrophobic chain in the membrane molecule (Ourisson \& Nakatani 1999). However, nature did it with three carbon atoms in the central chiral unit and with two hydrophobic chains linked to the amphiphilic head group: all of which can be synthesized from D-GLA (see below). If one follows the ideas that homochirality is needed for the spontaneous self-assembly of cell-membranes and that homochirality has originated from a pool of simple biomolecules, why would the spontaneous synthesis not have chosen the simplest track and synthesized simpler homochiral membrane molecules than diclycerides, phosphoro-lipids and phosphoro-ethers? However, the first chiral component in the formose reaction is GLA from which D-GLA-3phosphate and all of the different membrane molecules can be synthesized.

A central question about the biological evolution is "what came first, the metabolism or the replication?' (Pross 2004). Glycolysis is common for all living systems. Replication, however, is a very complex process where one of the stages (the last?) is the creation of ensembles of cells with cytokinesis. The fact that the membrane molecules in Archaea are fundamentally different from other membrane molecules with respect to homochirality and with no common origin of an enzyme for their synthesis but that all living systems contain glycolysis points toward evolution starting with establishing metabolism and some biological (replication) complexity before the cells or primitive vesicles were created with their diversity. This hypothesis is supported by an extensive amino acid sequence analysis (Fukuchi and Otsuka 1996) with the conclusion that the evolution towards biomembrane synthesis may be positioned as an event following the establishment of a section of the glycolic pathway from D-GLA-3phosphate.

The present hypothesis for the early self-assembly and establishment of homochirality is that the first step was the establishment of homochiral domains of D-GLA at the surface and within the droplets of amphiphilic and lipophilic molecules in the emulsion. In order to synthesize D-GLA-3phosphate, one needs a phosphorylation. However, D-GLA3-phosphate is soluble in water under alkaline conditions because it is charged, and the creation of charged phosphoroderivatives of D-GLA would make them soluble and ensured an aqueous environment of D-GLA-3-phosphate. In order to synthesize either sn-glycerol-1-phosphate or sn-glycerol-3-phosphate from D-GLA, one needs a mild reduction and a phosphorylation. Depending on whether one synthesizes the phosphoro-ester before or after the reduction one obtains either sn-glycerol-1-phosphate or a mixture of sn-glycerol-1-phosphate and sn-glycerol-3-phosphate (and other products). This is shown in Fig. 5. Apparently nature has spontaneously done both, and the aqueous environment must has contained both ingredients for the synthesis and self-assembly of the homochiral membranes in Archaea and Prokaryotes. Thus, the surface of D-GLA in the micelles could have served as both the fluid template for stereospecific synthesis of biomolecules as well as the source for synthesis of the central molecules in the metabolism and different membranes.

The formation of a homochiral environment of D-GLA also ensured a world of L-amino acids: L-Serine is observed to make complexes with D-GLA as well as with other Lamino acids in aqueous solutions (Kock et al. 2002; Takats et al. 2003) whereby D-GLA and L-Serine establish the stereospecific link between D-sugars and L-amino acids, and the surface of homochiral D-GLA could also be the fluid template for the synthesis of simple stereospecific peptides.

The hypothesis is that homochirality is obtained as a spontaneous reaction in emulsions of complex mixtures of amphiphilic and lipophilic bio-molecules as a precondition for biological evolution. The homochirality of D-GLA in an emulsion at the hydrothermal reactors would have ensured a coherent aqueous environment in which the stereospecific self-assembly was controlled by D-GLA. Glyceraldehyde fulfils the physico-chemical and biochemical conditions for having performed such a symmetry break by self-assembling with active isomerization kinetics. However, it remains to be demonstrated that one indeed can obtain homochirality under such conditions.

\section{References}

Amend, J.P. \& Shock, E.L. (2001). Energetics of overall metabolic reactions of thermophilic and hyperthermophilic archea and bacteria. FEMS Microbiol. Rev. 25, 175-243.

Avalos, M., Babiano Cintas, P., Jimenez, J.L. \& Palacios, J.C. (2004). Symmetry break by spontaneous crystallization - is it the most plausible source of terrestrial handedness we have long been looking for? - a reappraisal. Origin Life Evol. Biosphere 34, 391-405.

Bada, J.L. (1972). Kinetics of racemization of amino acids as a function of pH. J. Amer. Chem. Soc. 94, 1371-1373.

Breslow, R. (1959). On the mechanism of the formose reaction. Thetrahedron Lett. 1, 22-26.

Butlerow, A. (1861). Formation synthetique d'une substance sucree. C. R. Acad. Sci. 53, 145-147.

Davis, B.K. (1998). The forces driving molecular evolution. Prog. Biophys. Mol. Biol. 69, 83-150.

Fedoroňko, M. \& Königstein, J. (1969). Kinetics of mutual isomerization of trioses and their dehydration to methylglyoxal. Coll. Czech. Chem. Commun. 34, 3881-3894.

Fukuchi, S. \& Otsuka, J. (1996). Evolution of the self-reproducing system to the biosynthesis of the membrane: an approach from the amino acid sequence similarity in proteins. J. Theor. Biol. 182, 117-136.

Gabel, N.W. \& Ponnamperuma, C. (1967). Model for origin of monosaccharides. Nature 216, 453-455. 
Gresham, W.F. \& Grigsby, W.E. (1949). An improved synthesis of DL-glyceraldehyde. J. Org. Chem. 14, 1103-1107.

Kock, K.J., Gozzo, F.C., Nanita, S.C., Takats, Z., Eberlin, M.N. \& Cooks, R.G. (2002). Chiral transmission between amino acids: chirally selective amino acid substitution in the serine octamer as a possible step in homochirogenesis. Angew. Chem. Int. Ed. 41, 1721-1724.

Koga, Y., Kyuragi, T., Nishihara, M. \& Sone, N. (1998). Did archaeal and bacterial cells arise independently from noncellular precusors? A hypothesis stating that the advent of membrane phospholipid with enantiomeric glycerophosphate backbones caused the separation of the two lines of descent. J. Mol. Evol. 46, 54-63.

Kondepudi, D.K., Kaufman, R.J. \& Singh, N. (1990). Chiral symmetry breaking in sodium chlorate crystallization. Science 250, 975-976.

Leclercq, M., Collet, A. \& Jacques, J. (1976). Mesure de la stabilite des racemiques vrais. Tetrahedron 32, 821-828.

Martin, M. \& Russell, M.J. (2003). On the origins of cells: a hypothesis for the evolutionary transitions from abiotic geochemistry to chemoautotrophic prokaryotes, and from prokaryotes to nucleared cells. Philos. Trans. R. Soc. London Ser. B 358, 59-85.

Morgenlie, S. (1973). Oxidation of carbohydrate derivatives with silver carbonate on celite. Acta Chem. Scand. 27, 1557-1564.

Ourisson, G. \& Nakatani, Y. (1999). Origins of cellular life: molecular foundations and new approaches. Thetrahedron 55, 3183-3190.

Pasteur, M.L. (1850). Recherches sur les propriétés spécifiques des deux acides qui composent l'Acide racemique. Ann. Chim. Phys. 28, 56-99.

Peretó, J., López-García, P. \& Moreira, D. (2004). Ancestral lipid biosynthesis and early membrane evolution. Trends Biol. Sci. 29, 469-477.

Pross, A. (2004). Causation and the origin of life. Metabolism or replication first? Origin Life Evol. Biosphere 34, 307-321.

Russell, M.J. \& Martin, M. (2004). The rocky roots of the acetyl-COA pathway. Trends Biochem. Sci. 29, 358-369.

Schlesinger, G. \& Miller, S.L. (1983). Prebiotic synthesis in atmospheres containing $\mathrm{CH}_{4}, \mathrm{CO}$, and $\mathrm{CO}_{2}$. J. Mol. Evol. 19, 383-390.

Shock, E.L. (1992). Chemical environments of submarine hydrothermal systems. Origin Life Evol. Biosphere 22, 67-107.
Takats, Z., Nanita, C. \& Cooks, R.G. (2003). Serine octamer reactions: indicators of prebiotic relevance. Angew. Chem. Int. Ed. 42, 3521-3523.

Toxvaerd, S. (2000). Molecular dynamics simulations of isomerization kinetics in condensed fluids. Phys. Rev. Lett. 85, 4747-4750.

Toxvaerd, S. (2004a). Domain catalyzed chemical reactions: a molecular dynamics simulation of isomerization kinetics. J. Chem. Phys. 120, 6094-6099.

Toxvaerd, S. (2004b). Droplet formation in a ternary-fluid mixture: spontaneous emulsion and micelle formation. J. Phys. Chem. 108, 8641-8645.

Vik, J.-E. (1974). Base-catalyzed retro condensation reactions of some $\alpha$-hydroxymethyl carbonyl and nitro compounds. Acta Chem. Scand. B 28, 509-516.

Vladimirov, M.G., Ryzhkov, Y.F., Alekseev, V.A., Bogdanovskaya, V.A.O. \& Kritsky, M.S. (2004). Electrochemical reduction of carbon dioxide on pyrite as a pathway for abiogenic formation of organic molecules. Origin Life Evol. Biosphere 34, 347-360.

Washington, J. (2000). The possible role of volcanic aquifers in prebiologic genesis of organic compounds and RNA. Origin Life Evol. Biosphere 30, 53-79.

Weber, A.L. (1987). The triose model: glyceraldehyde as a source of energy and monomers for prebiotic condensation reactions. Origin Life 17, 107-119.

Weber, A.L. (1991). Origin of fatty acid synthesis: thermodynamics and kinetics of reaction pathways. J. Mol. Evol. 32, 93-100.

Weber, A.L. (1992). Prebiotic sugar synthesis: hexose and hydroxy acid synthesis from glyceraldehyde catalyzed by iron(iii) hydroxide oxide. J. Mol. Evol. 35, 1-6.

Weber, A.L. (1997). Prebiotic amino acid thioester synthesis: thioldependent amino acid synthesis from formose substrate (formaldehyde and glycolaldehyde) and ammonia. Origin Life Evol. Biosphere 28, 259-270.

Weber, A.L. (2001). The sugar model: catalysis by amines and amino acid products. Origin Life Evol. Biosphere 31, 71-86.

Weber, A.L. (2002). Chemical constraints governing the origin of metabolism: the thermodynamic landscape of carbon group transformation under mild aqueous conditions. Origin Life Evol. Biosphere 32, 333-357. 